\title{
Neurociências, neurocultura e autoajuda cerebral
}

ORTEGA, F. Neurosciences, neuroculture and cerebral self-help. Interface - Comunic., Saude, Educ., v.13, n.31, p.247-60, out./dez. 2009.

The aim of this paper was to analyze the phenomenon called neuroascesis or cerebral self-help, within the context of increasing impact of the neurosciences and the emergence of neuroculture and the cerebral subject. In order to accomplish this, it is important to understand the sociocultural context of neuroascesis, which corresponds to what is being called somatic culture or biosociality. The purpose of the article was to explore how a reductionistic form of subjectivity, the cerebral subject, enables the appearance of cerebral practices of the self, i.e. practices of how to act on the brain in order to maximize its performance. Such practices lead to the formation of new types of sociality.

Keywords: Neurosciences. Cerebral subject. Neuroculture. Cerebral self-help.
Este artigo pretende analisar o fenômeno denominado de neuroascese, ou autoajuda cerebral no contexto do crescente impacto das neurociências e do surgimento da neurocultura e do sujeito cerebral. Para tanto, é importante compreender o âmbito sóciocultural mais amplo no qual a neuroascese se insere e que corresponde ao que vem se chamando de 'cultura somática' ou, mais especificamente, de biossociabilidade. $\mathrm{O}$ objetivo do artigo é explorar como uma forma de subjetividade reducionista, o sujeito cerebral, dá lugar à aparição de práticas de si cerebrais, isto é, práticas de como agir sobre o cérebro para maximizar a sua performance, que levam a formação de novas formas de sociabilidade.

Palavras-chave: Neurociências. Sujeito cerebral. Neurocultura. Autoajuda cerebral.
${ }^{1}$ Instituto de Medicina Social, Universidade do Estado do Rio de Janeiro. Rua São Francisco Xavier, 524, pavilhão João Lyra Filho, $7^{\circ}$ andar, blocos D e E. Maracanã, Rio de Janeiro, RJ, Brasil. 20.550-900

fjortega2@gmail.com 


\section{Da bioascese à neuroascese}

Este texto pretende analisar o fenômeno que denomino neuroascese, ou autoajuda cerebral no contexto do crescente impacto das neurociências e do surgimento da neurocultura e do sujeito cerebral. Para tanto, é importante compreender o âmbito sociocultural mais amplo no qual a neuroascese se insere na nossa cultura contemporânea, e que corresponde ao que vem se chamando de 'cultura somática' (Ortega, 2008), 'indivíduos somáticos'2 ou, mais especificamente, de biossociabilidade. Neste trabalho, vou me servir desse conceito em um sentido mais amplo que o de Paul Rabinow (1996), que o cunhou para analisar as implicações socioculturais e políticas da genética e do projeto Genoma. Para o antropólogo americano, a genética deixará de ser apenas uma metáfora biológica para descrever o social, levando à formação de identidades e práticas sociais. No texto, uso esse conceito de Rabinow, sublinhando o elemento dos processos de subjetivação, mas sem limitá-lo especificamente à genética, estendendo o uso a diferentes formas de subjetividade biológica ou somática presentes nas sociedades contemporâneas, incluindo o sujeito cerebral, ao qual me refiro mais adiante.

Entendo por biossociabilidade uma forma de sociabilidade ou de coletividade constituída por grupos de interesses privados, não mais reunidos segundo critérios de agrupamento tradicional como classe, estamento, orientação política, como acontecia na biopolítica clássica analisada por Foucault ${ }^{3}$, mas segundo critérios de saúde, performances corporais, doenças específicas, estatuto genético compartilhado por outros indivíduos, longevidade, entre outros. Narrativas de experiência de doenças e performances do sofrimento que aparecem na mídia, bem como a disseminação e moralização do vocabulário médico-fisicalista baseado em constantes biológicas, taxas de colesterol, tono muscular, desempenho físico ou capacidade aeróbica fornecem os critérios de avaliação individual e destacam a existência corporal do self como lugar privilegiado de construção de relações consigo e com os outros nas sociedades biomédicas contemporâneas (Ortega, 2008; Rose, 2007). Coletividades organizadas em torno de classificações biomédicas ocupam cada vez mais espaço, podendo reivindicar uma influência maior na tomada de decisões acerca de terapias apropriadas para doenças específicas; obter fundos para a pesquisa, ou marcar distância da própria investigação biomédica e contestar o estatuto nosológico de determinadas doenças, como vemos no caso do autismo.

Em sociedades fortemente marcadas pelas teorias e práticas psicanalíticas, como o caso do Brasil e dos EUA durante as sete primeiras décadas do século passado, era frequente que os indivíduos se descrevessem como habitados por um espaço interior e psicológico, fonte de desejos e lugar de escrutínio de todos os transtornos psíquicos, avaliando a si mesmo e aos outros e agindo sobre si mesmo com base nessa crença. Os discursos psicológicos possibilitaram formas de relacionar-se consigo mesmo em termos de neuroses, desejos inconscientes, trauma, e repressão, dando uma centralidade à sexualidade na definição da vida psíquica. Nas últimas décadas, porém, esse espaço interno característico da cultura psicológica fortemente influenciada pela psicanálise vem sendo achatado e, em alguns casos, deslocado por uma localização de doenças e transtornos no corpo e no cérebro (Ortega, 2008). Sirva, como exemplo, o caso do alcoolismo: Segundo os 12 passos dos Alcoólicos Anônimos (AA), deixar de beber corresponde a uma transformação interior. O alcoolismo é visto como uma fraqueza moral e da vontade, uma falha localizada no interior do self - o indivíduo deve reconhecer que é, e sempre será, um alcoolista e que deve trabalhar para estabelecer níveis de introspecção e disciplina que permitam deixar o vício de maneira definitiva. Nas

\author{
${ }^{2}$ Nikolas Rose (2007, \\ p.26) define os \\ 'indivíduos somáticos' \\ como "aqueles seres \\ cuja individualidade é, \\ em parte pelo menos, \\ localizada na nossa \\ existência carnal, \\ corporal e que \\ experienciam, articulam \\ julgam e agem sobre si \\ mesmos em parte na \\ linguagem da \\ biomedicina".
}

\author{
${ }^{3} \mathrm{O}$ biopoder clássico, \\ descrito por Foucault, se \\ articulava como uma \\ anátomopolítica do \\ corpo e uma biopolítica \\ das populações. A \\ biopolítica foucaultiana \\ $(1999,1997,1976)$ \\ enfatizava, \\ especialmente, as \\ noções de sexualidade, \\ raça e degenerescência \\ cujo objetivo era a \\ otimização da qualidade \\ biológica das populações \\ e estava vinculada à \\ afirmação da burguesia \\ como classe social e ao \\ fortalecimento do Estado \\ Nacional. Diversos \\ autores têm estendido o \\ uso do bipoder e \\ biopolítica para o \\ contexto \\ contemporâneo. Neste \\ artigo uso o termo \\ biossociabilidade para \\ frisar as descontinuidades \\ com o biopoder clássico. \\ Para uma importante \\ crítica do uso do \\ conceito de bipoder em \\ autores como Negri e \\ Agamben, ver Rabinow \\ e Rose (2006). Para o \\ uso do termo \\ biossociabilidade em \\ diferentes contextos \\ empíricos, incluindo uma \\ reflexão atualizada de \\ Rabinow, ver a \\ interessante coletânea \\ de Gibbon e Novas \\ (2008).
}


descrições neurocientíficas do alcoolismo, em contrapartida, o objetivo das intervenções é o estado neuroquímico do cérebro. A bebida ativa o sistema de recompensas no cérebro, mas, em alguns indivíduos, o cérebro recompensaria a bebida de uma maneira desmesurada provocando desejos intensos pelo álcool. Trata-se de um sistema endorfínico superestimulado. As complexas práticas confessionais dos rituais dos AA são substituídas por simples decisões acerca de tomar ou não a medicação (Vrecko, 2006). Esse é um exemplo emblemático do deslocamento de explicações centradas na vida psíquica para as baseadas em parâmetros cerebrais.

No processo de descrição da individualidade e subjetividade em termos corporais, o cérebro ocupa um lugar privilegiado. O espetacular progresso das tecnologias neurocientíficas, o intenso processo de popularização, pela mídia, de imagens e informações que associam a atividade cerebral a praticamente todos os aspectos da vida, e certas características estruturais da sociedade atual vêm produzindo, no imaginário social, uma crescente percepção do cérebro como detentor das propriedades e autor das ações que definem o que é ser alguém. Ele responde, cada vez mais, por tudo aquilo que outrora nos acostumamos a atribuir à pessoa, ao indivíduo, ao sujeito. Inteiro ou em partes, o cérebro surgiu como o único órgão verdadeiramente indispensável para a existência do selfe para definir a individualidade.

Crenças, desejos e comportamentos são frequentemente descritos em um vocabulário cerebral ou neuroquímico. Nikolas Rose (2007) define este processo usando o termo 'self neuroquímico' (neurochemical self), isto é, a formação neuroquímica da pessoa. A emergência de 'neuroessencialismo', 'neurorrealismo' e 'neuropolítica' vem sendo ressaltada nas interpretações populares da neurociência (Racine, Bar-Ilan, Illes, 2005), resultando em um 'entusiasmo aparentemente acrítico' (Illes, Racine, 2005) diante dos avanços neurocientíficos. Acredito que a noção de "sujeito cerebral" (Vidal, 2009; Ortega, Vidal, 2007; Ehrenberg, 2004) resume adequadamente a redução da pessoa humana ao cérebro, que perpassa diferentes análises críticas da neurocultura contemporânea: a crença de que o cérebro é a parte do corpo necessária para sermos nós mesmos, no qual se encontra a essência do ser humano, ou seja, a identidade pessoal entendida como identidade cerebral.

Uso o termo 'sujeito cerebral' como uma derivação da noção de sujeito que Foucault (1984a, 1984b, 1976) analisa em sua história da subjetividade. Trata-se de uma categoria histórica e cuja ênfase recai precisamente nas formas e nos processos de subjetivação e nas tecnologias do self usadas pelos indivíduos para a formação de diferentes "subjetividades". Existiriam, assim, diferentes formas de subjetividade, tipos de "sujeito" na história da subjetividade, entre elas, o "sujeito cerebral" das sociedades contemporâneas. O sujeito cerebral não possui uma realidade prévia a suas corporificações performativas. Em outras palavras, o processo de subjetivação tem uma preeminência ontológica e, por isso, a ênfase recai nas noções, práticas e contextos pelos quais os seres humanos se tornam sujeitos cerebrais. Sujeitos cerebrais se formam e são formados mediante tecnologias do self sustentadas, em parte, pelo conhecimento especializado e sua divulgação pela mídia e pela cultura popular. Deve ser observado, porém, que o sujeito cerebral não é uma figura antropológica nem monolítica, nem hegemônica, e que os indivíduos não se subjetivam de uma única maneira. Existe uma coexistência entre ontologias cotidianas, que faz com que as pessoas mudem os registros na sua forma de agir, experienciar, pensar e falar de si mesmo e dos outros. Em determinados contextos podem recorrer a um vocabulário psicológico, em outros, ao cerebral ou neuroquímico. Diferentes ontologias do self, da psicanalítica à cerebral, convivem de maneira mais ou menos harmônica quando falamos de nós mesmos e vivemos nossas vidas.

No âmbito da biossociabilidade, o sujeito cerebral dá lugar à aparição de práticas de si cerebrais, as neuroasceses, isto é, discursos e práticas de como agir sobre o cérebro para maximizar a sua performance, que levam à formação do que vou chamar de neurossociabilidades e neuroidentidades. Elas constituem formas de "selves objetivos", de "autoconstituição objetiva" (objective self-fashioning), usando a expressão de Joseph Dumit (2004) para se referir ao processo de formação de um self objetivo, ou seja, uma categoria de pessoa desenvolvida mediante conhecimento especializado. É um processo duplo: por um lado, as práticas da ciência da medicina e da tecnologia formam selves mediante a experimentação científica, os exercícios de taxonomia médica, entre outros. Isto é, produzem "fatos" que definem objetivamente quem somos; por outro, os indivíduos formam seus próprios modelos de self a partir dos fatos científicos. A noção de self objetivo remete a uma 
Parkinson, esclerose múltipla, Transtorno de Déficit de Atenção e Hiperatividade (TDAH), demência Frontotemporal (Frontotemporal Dementia - FTD), Doença de Huntington (Hungtinton Disease), entre outros. As funções e objetivos desses grupos são diferentes, atendendo também as especificidades das diferenças doenças e transtornos, contemplando atividades que vão desde palestras e troca de informação e experiências até exercícios, serviços religiosos ou ajuda psicológica. Intervenções psicossociais diversas e troca de experiências podem acontecer em encontros regulares ou por meios virtuais, como internet, com o uso de videoconferências, comunidades no Yahoo, orkut, blogs e outros (A lista de organizações e de grupos de autoajuda nos diferentes países e continentes é enorme. Basta procurar em qualquer mecanismo de busca na internet).

d) O movimento da neurodiversidade: Os participantes desse movimento acreditam que uma conexão neurológica atípica não é uma doença que deva ser tratada, e sim uma diferença humana que deve ser respeitada. Indivíduos diagnosticados com autismo de 'alto-funcionamento' (geralmente associado com a chamada Síndrome de Asperger) impulsionam o movimento. Para eles, o autismo é uma parte constitutiva de sua identidade. Uma 'cultura autista' vem sendo desenvolvida segundo modelos de pensamento e estilos de vida considerados autistas, como se depreende dos numerosos websites existentes na internet sobre o tema. Neles encontramos desde dicas de literatura que trata de autismo até listas de organizações de apoio a autistas e potenciais parceiros para casamento autistas.

A noção de neurossociabilidade permite compreender como o sujeito cerebral se torna um critério de agrupamento, isto é, como dá lugar à formação de diversos grupos, sejam eles de portadores de alguma doença neurodegenerativa e seus familiares, ou de indivíduos que se reúnem para testar a sua performance cerebral, ou de grupos de autistas que usam o critério cerebral para reclamar um acesso à cidadania que o modelo biomédico Ihes tinha negado. Um modelo de "subjetivade-objetiva", como em um oxímoro, que surgiu a partir de uma ideologia reducionista e solipsista da compreensão da subjetividade e da vida social, serve paradoxalmente para criar novas formas de subjetividade e de sociabilidade que têm no cérebro a sua âncora. Obviamente, existe uma diferença do ponto de vista do significado sociopolítico entre grupos de apoio a portadores de doenças e transtornos específicos, grupos pró e anticura, que aparecem no debate em torno do autismo, por um lado, e neurocomunidades de adolescentes, clubes do cérebro e empresários dos neuronegócios, pelo outro. Ambos os grupos têm o cérebro como referência. Todavia, enquanto os primeiros poderiam ser comprendidos como formas de resistência a um tipo de racionalidade política neoliberal aparelhada com tecnologias neurocientíficas, os segundos se enquadram plenamente nos objetivos dessa racionalidade política.

Enfim, o mapeamento da neurossociabilidade está apenas começando e é um terreno em constante expansão. Vou me concentrar, a seguir, no resto do texto, em alguns aspectos dessa neurossociabilidade e das práticas de si cerebrais.

\section{Plasticidade cerebral e neuronegócios}

Convém, em primeiro lugar, fazer uma pequena referência ao conceito de plasticidade cerebral (Schwartz, Begley, 2002; Jones, 2000), tomado como ponto de partida pelas diversas práticas neuroascéticas. Tornou-se senso comum falar de plasticidade cerebral. Já, em 1890, no capítulo dedicado ao 'Hábito' em The Principles of Psychology, William James observou que o hábito era produzido pela plasticidade cerebral: os hábitos deixariam marcas, sulcos na estrutura neural dos indivíduos. A nossa experiência deixa marcas no cérebro (James, 1952). O achado do filósofo americano foi comprovado empiricamente por Graham Brown e Charles Sherrington (1912) ao descobrirem, pesquisando com macacos, que as representações corticais dos movimentos não são inatas e refletem a história do uso do sistema motor. Trabalhos como os de Ivory Franz (1915) e de Karl Lashey (1923) contribuíram para que, por volta de 1950, existisse um corpo de evidência razoável de que o córtex cerebral é dinâmico, remodelado continuamente pela experiência.

Apesar das evidências, o paradigma do cérebro 'imutável' tornou-se predominante, relegando ao esquecimento os achados de Sherrington, Frank e Lashey, entre outros. A partir da década de 1990, surge um número crescente de trabalhos que pretendem demonstrar como a experiência produz 
alterações no cérebro (Merzenich, Jenkins, 1994). Tenta-se comprovar as alterações cerebrais causadas por hábitos, decisões conscientes, atos de vontade, práticas de atenção, exercício físico, alimentação, práticas de meditação, entre outros.

Cada vez mais pesquisadores relatam a descoberta (que já foi apontada por James no final do século $\mathrm{XIX)} \mathrm{de} \mathrm{que,} \mathrm{ao} \mathrm{contrário} \mathrm{do} \mathrm{que} \mathrm{se} \mathrm{acreditava,} \mathrm{as} \mathrm{conexões} \mathrm{entre} \mathrm{células} \mathrm{nervosas} \mathrm{do} \mathrm{cérebro} \mathrm{criadas}$ na infância não se mantêm inalteradas durante toda a vida adulta do indivíduo. Tornou-se um 'dado empírico' (como se depreende da avalanche de artigos sobre o tema) que a estrutura e o funcionamento do cérebro pode modificar-se até idade bem avançada, e novos neurônios são criados (Schwartz, Begley, 2002; Jones 2000; Weiller, Rijntjes, 1999). É na base desse conhecimento empírico que tem acontecido um verdadeiro 'boom' de práticas neuroascéticas na última década, que deram lugar a um florescente mercado de neurobusiness em constante expansão (Woldbring, 2007). Por exemplo, pesquisadores descobriram recentemente que as atividades aeróbicas possuem benefícios importantes, para além dos cardiovasculares e da utilidade no tratamento da depressão, já conhecidos desde algum tempo. O exercício encoraja os cérebros saudáveis a funcionarem a níveis ótimos, permitindo aumentar sua performance e promovendo a plasticidade cerebral. Também promete retardar a progressão da Alzheimer e os sintomas do Parkinson. Os mesmos efeitos foram constatados recentemente em relação à alimentação saudável, com baixo teor de gorduras saturadas e rica em Ômega 3 (Brownlee, 2006a; Cotman e Berchtold, 2002). A idéia básica por trás dessas pesquisas é muito simples, "cuidando de seu corpo, seu cérebro também se beneficia", observa Carol Greenwood (apud Brownlee, 2006a), pesquisadora em nutrição e envelhecimento da Universidade de Toronto. Essa afirmação exemplifica a tendência recorrente de um 'neurosolipsismo' que leva a confundir ou reduzir o todo às partes, o corpo ao cérebro: embora as práticas treinem o corpo, se afirma que é o cérebro que está sendo visado.

Tomando como base os novos conhecimentos sobre a plasticidade cerebral, surgiu, em San Francisco, a partir do trabalho pioneiro do Dr. Michael Mezenich, membro da academia das ciências e neurocientista renomado, a empresa Posit Science Corporation, uma das diversas empresas que estão aparecendo no ramo dos neuronegócios (Lynch, 2004). O objetivo de Posit Science é "ajudar as pessoas a prosperar ao longo de suas vidas. Fazemos isso fornecendo ferramentas efetivas e não invasivas que comprometam a plasticidade natural do cérebro na tarefa de melhorar a saúde cerebral" (Teichert, 2005). O programa de fitness cerebral de Posit Science focaliza no aperfeiçoamento da velocidade, precisão e intensidade com as quais nosso cérebro recebe, registra e lembra aquilo que ouvimos. O programa cerebral é o primeiro passo; a empresa planeja oferecer uma 'academia cerebral' (brain gym) completa para treinar os diferentes sistemas cognitivos: visão, controle executivo, equilíbrio, mobilidade.

A publicidade da empresa lembra a do marketing de produtos de beleza: o programa de neuroascese promete 'rejuvenescer' a plasticidade 'natural' do cérebro, adiando o 'declínio' mental até em dez anos. Não por acaso, o principal alvo de Posit Science é a população idosa. Mesmo que o 'Brain Gym' não represente a fonte da juventude, Mezernich afirma que é "parte da solução", pois terá um grande impacto não apenas na qualidade de vida dos idosos. E, o que é mais importante, promete "manter a vitalidade, o vigor das pessoas". Vitalidade e vigor necessários para que a "longevidade cerebral' (brainspan) possa acompanhar a longevidade corporal (lifespan) em constante aumento (Anônimo, 2006). Da mesma maneira que a fitness corporal, a fitness cerebral exige uma grande dose de disciplina, vontade e automotivação. A recompensa é grande, nos promete "inverter o processo de envelhecimento cerebral" (Olney, 2006). Por outro lado, os neuronegócios são promissórios. Posit Science prevê um potencial de venda enorme considerando que a metade dos norte-americanos que atingirem 85 anos sofrerão de alguma forma de demência. E os preços são salgados, começando em torno dos U\$500 (Olney, 2006).

\section{Autoajuda cerebral}

As descobertas da neuroplasticidade têm dado lugar a um mercado de best-sellers de ginástica e autoajuda cerebral com embasamento científico diverso. Eles prometem desenvolver determinadas regiões do cérebro (especialmente o hemisfério direito, tradicionalmente reprimido e negligenciado), 
permitindo desde aumentar a performance do raciocínio e da memória, combater depressão, ansiedade, adições e compulsões diversas, até melhorar a performance sexual, atingir a felicidade ou estabelecer um contato direto com Deus. Essa literatura constitui uma amálgama de trabalhos diversos que inclui: estudos mais sérios de neurocientistas, psicólogos cognitivos e psiquiatras renomados que, baseados nas novas descobertas, oferecem um programa de exercícios para aumentar a performance e o poder cerebral, prevenindo a decadência mental e combatendo demências. Habilidades tais como percepção, memória de curto e longo tempo, habilidades lógicas, verbais e visuoespaciais podem ser desenvolvidas com a ajuda das práticas neuroascéticas (Goldberg, 2001; Chafetz, 1992; Goldman, Klatz, Berger, 1991; Mark, Mark, 1991; Winter, Winter, 1987).

Outros best-sellers, escritos geralmente por autores de autoajuda que descobriram o filão da neuroascese, vão muito mais longe nos resultados atingíveis com seus programas de autoajuda cerebral, apesar de afirmarem possuir embasamento científico. Eles prometem: identificar significados ocultos nas conversas das pessoas; absorver fatos como esponjas e reproduzi-los palavra por palavra anos depois; ler e compreender um livro em trinta minutos; ou gravar, na memória, fatos, imagens e até livros completos. Entre eles, os mais voltados para movimentos de New Age, e usando um vocabulário científico e um linguajar técnico, acreditam poder manipular o cérebro para atingir qualquer resultado desejável. Esses autores recorrem, com frequência, a simplificações 'chulas' da mecânica quântica para afirmar que a realidade é uma ilusão criada por nosso cérebro e que o "universo é a mente e a mente é o universo" (Spotts, Atkins, 1999, p.80). Os exercícios garantem promover estados alterados da consciência capazes de conectar o cérebro com as forças do universo e com uma inteligência superior, a Mente cósmica ou Divina. O que resulta surpreendente desses últimos best-sellers é que reproduzem todos os tópicos da literatura da autoajuda tradicional usando um vocabulário cientificista e coisificador.

A partir da segunda metade da década de 1980, autores como Antonhy "Tony" Robbins recuperam o credo básico da tradição da clássica Self-Help americana, dos movimentos oitocentistas de New Thought, e da Christian Science (Fuller, 2001). Isto é, a ideia do poder da mente sobre a matéria, chamada doravante de Mind-Power e que pressupõe o poder do cérebro sobre a mente. Essa visão do self é retraduzida em uma linguagem pseudocientífica, como um self cibernético que pode ser reprogramado para obter a máxima performance pessoal (McGee, 2005). Já no início dos anos sessenta, o cirurgião plástico nova-iorquino Maxwell Maltz descobriu um filão ao vender o velho Mind-Power oitocentista com uma roupagem cibernética, dotando-o de legitimidade científica. O resultado, chamado de "programação neurolinguística" (neurolinguistic programming), propunha que crenças e desejos seriam uma espécie de software que pode ser instalado no hardware, o cérebro humano. A receita foi vendida, em 1960, como Psycho-Cybernetics, vendendo até a atualidade mais de trinta milhões de exemplares no mundo inteiro (McGee, 2005; Maltz, 1960). Mesmo autores como Shirley McLaine lançam mão de um vocabulário cientificista e, na busca de legitimidade, falam de forças, vibrações, eletromagnetismo, hemisférios cerebrais (Fuller, 2001; Green, 1992).

"A novidade" da autoajuda cerebral, escreve Rüdiger (1995, p.120), "é o estágio avançado de reificação da subjetividade, que se transferiu dos mecanismos do subconsciente para as circunvoluções do córtex cerebral". Todos os lugares comuns da autoajuda tradicional estão presentes numa roupagem cientificista e cerebralizada. Vale mencionar: a ênfase na criatividade que engendra a realidade; a ideia de um 'eu interior' que pode ser cultivado e promovido pela manipulação cerebral; a insistência na autonomia e autocontrole de nossos destinos e até da própria realidade, atingida agora com práticas neuroascéticas. A absoluta irredutibilidade da categoria de responsabilidade e autonomia individual, enfatizando controle e autodomínio, bem como a redutibilidade total da realidade aos desígnios do pensamento são também realçadas na autoajuda cerebral. Finalmente, a total obsolescência do outro, do ambiente social e cultural no eu cerebral construído pelas práticas neuroascéticas. Se na autoajuda tradicional era a mente o centro definidor do sujeito, e seu poder permitia mudar a vida, realizar nosso desejo e monitorar nosso desempenho, agora, o cérebro ocupa esse lugar reservado outrora à mente. $\mathrm{O}$ velho slogan "você é o que for sua mente", aparece agora em uma versão cerebralizada: "você é o que for seu cérebro".

Um tópico importante da autoajuda tradicional, a ideia da mente dividida e em luta - em que uma parte é insubmissa e deve ser controlada, ou é subutilizada - remete à tradição do cérebro duplo de 
Arthur Wigan e outros, tal como descrita pela historiadora das neurociências Anne Harrington (1987). Na época pós-Broca, isto é, na época posterior à descoberta da localização da faculdade da linguagem no hemisfério esquerdo do cérebro pelo neurologista francês, Paul Broca, o hemisfério esquerdo era considerado superior, ao ser responsável pelas atividades intelectuais e civilizadas, predominando em homens, brancos e europeus. O direito, em contrapartida, preponderava em mulheres, criminosos, índios, negros, loucos e homossexuais (Harrington, 1987). Os best-sellers de autoajuda cerebral reproduzem e exploram o 'boom' do cérebro direito, que tem lugar a partir dos anos 60 (invertendo a tradicional valorização do hemisfério esquerdo), no contexto da contracultura e das pesquisas sobre o 'cérebro dividido' (split-brain) de Bogen, Gazzaniga e Sperry, entre outros (Harrington, 1987; Gazzaniga, 2005, 1985, 1973; Harris, 1985, 1980; Blakeslee, 1980; Bogen, 1977, 1973; Gazzaniga, Bogen, Sperry, 1962). Boa parte dos bestsellers de autoajuda cerebral embarca na esteira da reabilitação do hemisfério direito. Um marco importante nessa tendência é a publicação, em 1972, do clássico frequentemente citado The Psychology of consciousness de Robert Ornstein (1972). Nele, o racionalismo e tecnificismo da sociedade ocidental, com sua ênfase na lógica e na linguagem, patente no predomínio do hemisfério esquerdo, teria reprimido as tendências holísticas e místicas do direito, mais próximo do pensamento oriental ${ }^{6}$. O mercado de autoajuda é inundado por uma enxurrada de títulos ligando o hemisfério direito aos mais bizarros fenômenos, desde a tipificação de artistas, músicos, políticos e ditadores, segundo sua 'orientação' cerebral, até a sexualidade tântrica, a capacidade mediúnica e outras atividades paranormais próprias do cérebro direito (Capacchione, 2001; Spotts, Atkins, 1999; Wells, 1989; Ehrenwald, 1984).

Especialmente na pedagogia aparece, desde finais dos anos 60, toda uma moda neuroeducativa que insiste nas inúmeras vantagens escolares do treinamento do cérebro direito, repudiando o sistema educativo tradicional baseado no hemisfério esquerdo (Herman, 1981; Edwards, 1979; Gainer, Gainer, 1977; Hunter, 1976). Os novos neuroeducadores propõem como solução um equilíbrio hemisférico no currículo que compense os excessos da educação baseada no hemisfério esquerdo com seus fracassos educativos:

\begin{abstract}
Os estudantes que não acham a escola muito empolgante talvez possuam uma orientação cerebral esquerda. Para eles, muitas tarefas para o cérebro esquerdo não fazem sentido. Existem evidências de que os pobres nas grandes cidades tendem a possuir uma orientação cerebral direita, enquanto que indivíduos de classe média são mais orientados para o hemisfério esquerdo. Se isso for verdade, explicaria por que muitos pobres nas cidades não têm sucesso nas escolas e por que consideram irrelevantes muitas tarefas exigidas na escola. (apud Ornstein, 1997, p.92)
\end{abstract}

Os educadores não aprenderam novos desenvolvimentos a partir da literatura primária, mas predominantemente de livros e revistas que popularizam esse conhecimento, simplificam e reconstroem muitos dos achados científicos, conduzindo a uma visão simplificada, tosca, e idealizada (Harris, 1985).

No contexto de uma genealogia das práticas de si cerebrais, não se trata de separar o joio do trigo, as evidências científicas da charlatanice e das afirmações e propostas disparatadas, por mais importante que seja essa tarefa ${ }^{7}$. Mais relevante é mostrar como as práticas neuroascéticas reproduzem a lógica do sujeito cerebral e da autoajuda tradicional com uma roupagem cientificista. Ao nível das práticas de

\author{
${ }^{6}$ Em um livro de 1997, \\ The Right Brain: Making \\ sense of the \\ Hemispheres, Ornstein \\ faz uma certa mea-culpa \\ ao se perguntar em \\ relação aos estudos sobre \\ o hemisfério direito: \\ "Como é possível que as \\ coisas escaparam do \\ controle? Como é \\ possível que todas essas \\ pessoas tão bem \\ intencionadas se \\ afastaram tanto da \\ evidência e inclusive do \\ trabalho científico? Tudo \\ começou com boas \\ idéias, boas intenções e \\ bons trabalhos" \\ (Ornstein, 1997, p.88).
}

\footnotetext{
${ }^{7}$ Os próprios usuários dos diversos programas de brain-fitness não parecem se preocupar pelo seu embasamento científico. "Surpreende-me que as pessoas não se preocupem mais com a ciência", observa Torkel Klinberg, professor de neurociência cognitiva do Instituto Karolinska de Estocolmo, "que não perguntem, 'onde está a evidência de que esses programas funcionam?'" (Lawton, 2008, p.29).
} 
si, muitos motivos são recuperados da tradição neuroeducativa da frenologia e da autoajuda tradicional do século XIX. É surpreendente constatar como os programas neuroascéticos dos best-sellers contemporâneos repetem recomendações sobre exercícios cerebrais e sobre a importância do exercício físico, da dieta equilibrada e do efeito de álcool, drogas e outras toxinas no cérebro de autores oitocentistas, tais como John Harvey Kellog, no seu First Book in Physiology and Hygiene (1887). Mas os contextos socioculturais e a finalidade das práticas são diferentes. No fim do século XIX, o cérebro 'azeitado' de Kellog remete a um contexto de debilitamento e deslegitimação das regras sociais e da ordem moral, no qual o corpo físico, social e político estava fora de controle, só recuperável pela vontade individual e pelas práticas bio - e neuroascéticas. A fitness cerebral destinava-se a recuperar a retidão moral e garantir a ordem sociomoral erodida pela perda da legitimidade da autoridade tradicional (Gunsfield, 1992). Hoje, em contrapartida, o sujeito cerebral não pretende restaurar ou legitimar formas tradicionais de autoridade, e sim, inserir-se nas demandas da maximização da performance corporal da cultura somática na govermentalidade neoliberal. No entanto, mesmo existindo descontinuidades nos contextos socioculturais e nos objetivos dessas práticas, no nível das práticas de si, isto é, da (neuro)ascese, existem assombrosas semelhanças. Trata-se dos mesmos exercícios, da mesma dietética cerebral, de maneiras semelhantes de potencializar a performance cerebral, embora com finalidades diversas. Em ambos os casos, as práticas neuroascéticas redundam na formação de 'selves objetivos'. Para não cair em anacronismos e postular uma continuidade direta (o que, sem dúvida, seria ingênuo), é necessário, no entanto, guardar a devida distância entre os dois momentos históricos e levar em consideração diferentes fatores na formação de 'selves objetivos', a partir da segunda metade do século XX, que não existiam na época da frenologia e dos movimentos de Self-Help e de New Thought, tais como: o papel desempenhado pela mídia, as tecnologias de visualização médica, a indústria de psicofármacos, entre outros (Rose, 2007; Ehrenberg, 2004; Healy, 2002).

\section{Ginástica cerebral na cultura somática}

A genealogia da neuroascese permite constatar também como os discursos e práticas dos bestsellers de fitness cerebral repetem as metáforas e os discursos da fitness corporal predominantes na cultura somática da biossociabilidade. Já a própria ideia de 'fitness cerebral' ou 'neuróbica', presente no título da maioria desses livros (Cohen, Goldsmith, 2002; Eiffert, 1999; Dennison, Dennison, 1994, 1989; Goldman, Klatz, Berger, 1991; Mark, Mark, 1991; Winter, Winter, 1987), remete a essa analogia. Assim, "da mesma maneira que o levantamento de peso na academia ou a corrida fortalecem um determinado grupo de músculos, os exercícios mentais parecem fortalecer e aprimorar as funções cognitivas por longo tempo" (Tannen, 2005). Os diversos livros e programas falam de "academia cerebral" (Brain Gym)" e de "levantamento de peso mental" (mental weight lifting), (CBS, 2006). Os "músculos cerebrais" (Goldberg, 2001, p.255) devem ser treinados sem excesso para não causar "câimbras cerebrais" (brain cramps) (Chafetz, 1992, p.72). Os livros nos ensinam alguns "alongamentos cerebrais" (brain stretches) que "queimam algumas calorias sinápticas" (synaptic calories) para não nos tornarmos "batatas de sofá mentais" (mental couch potatoes) (Parlette, 1997, p.16), apesar de podermos usufruir da TV, verdadeiro "chiclete para o cérebro" (bubble gum for the brain) (Parlette, 1997, p.152-3). Diversos autores distinguem entre diferentes níveis de façanhas cerebrais, pois "você não precisa atingir o equivalente cerebral do nível de fitness física de Steffi Graf ou de Michael Jordan para ser mais rápido na conversa, melhor na resolução de problemas, possuir lembranças mais ricas e associações mais vivas" (Chafetz, 1992, p.23). Outros insistem em planejar um programa de treinamento cerebral sistemático, "para aqueles que desejam exercitar o cérebro sistematicamente como um atleta exercitaria vários grupos musculares" (Goldberg, 2001, p.252-3; Chafetz, 1992, p.213). Esse treinamento deve permitir que os diversos músculos cerebrais sejam exercitados alternadamente e de modo equilibrado. Para tanto, é importante ter um "marathon trainer" e manter "diários de malhação cerebral" (brain workout diaries) (Chafetz, 1992, p.213-4). Goldberg reconhece que a neuroascese "é uma extensão natural e lógica do exercício físico. A 'aptidão ou boa forma física' tornou-se um termo caseiro. A "aptidão ou boa forma cognitiva está a caminho de tornar-se a próxima tendência na cultura popular" (Goldberg, 2001, p.253). O sujeito cerebral transpôs o vocabulário da 
fitness corporal para o cérebro. Na cultura somática, fitness corporal e cerebral andam lado a lado. $\mathrm{Na}$ autoajuda cerebral os principais motivos da autoajuda tradicional aparecem em nova roupagem cientificista, temperados com o jargão da cultura somática e das academias de ginástica.

\section{Conclusão: sujeito cerebral, neuroascese e govermentalidade neoliberal}

É importante frisar que não podemos reduzir o surgimento e popularização das práticas de neuroascese descritas neste texto aos avanços dos conhecimentos neurofisiológicos, especialmente da plasticidade cerebral. Fazendo assim, negligenciamos o papel da ontologia do sujeito cerebral e da neurossociabilidade - e da cultura somática da biossociabilidade, em um sentido mais amplo. O sujeito cerebral implica formas de subjetivação, isto é, relações consigo e com os outros enquanto sujeitos cerebrais. Este fato remete a visões reducionistas e empobrecidas da vida subjetiva e relacional, segundo as quais o cérebro responde por tudo o que outrora costumávamos atribuir ao indivíduo, ao ambiente e à sociedade, com consequências severas em diversas esferas socioculturais e clínicas. Entre elas, o perigo das políticas identitárias reducionistas e as explicações da depressão e de outros transtornos e doenças mentais em termos exclusivamente cerebrais, fornecidas pela psiquiatria biológica aliada à indústria farmacêutica, ignorando-se os fatores ambientais e sociais. É preciso saber se queremos pagar esse preço.

A autoajuda cerebral deve ser inserida no contexto da genealogia da autoajuda, como foi sublinhado no texto. Trata-se de tecnologias de governamentalidade, de autogoverno. Na governamentalidade neoliberal, cada indivíduo é um empresário que deve administrar sua própria vida (Dean, 1999). Trata-se de uma forma de governo que demanda uma cidadania ativa e indivíduos capazes de monitorar e controlar a si mesmos. O sujeito autônomo, responsável e gestor dos próprios riscos, deve se adaptar e aprimorar suas capacidades e habilidades para atingir as demandas de um mercado cada vez mais competitivo. Nesse contexto, medidas neuroeducativas, aprimoramento cognitivo e outros tipos de práticas neuroascéticas se tornam moeda corrente, atingindo um caráter de quase obrigatoriedade numa sociedade que favorece selves ativos e emprendedores. A autoajuda cerebral se torna uma oferta e uma obrigação para o bem individual e da comunidade. Ela contribui na produção de cidadãos capazes e responsáveis por governar a si mesmo e aos outros. Ao mesmo tempo, práticas neuroascéticas contribuem para colocar essas subjetividades normativas no centro da sociedade neoliberal, permitindo ao estado e a outros atores governarem a distância e governarem mediante a liberdade dos indivíduos (Maasen et al., 2007). Se, segundo Foucault, todo dispositivo de saber-poder é um mecanismo de assujeitamento, mas, ao mesmo tempo, abre possibilidades da resistência, cabe a nós apostarmos em alternativas para esta ideologia simplificadora da vida subjetiva e relacional que não sejam epifenômenos do sujeito cerebral. 


\section{Referências}

ANÔNIMO. Retraining the brain: doctors test drug-free methods to restore lost mental capabilities. CBS NEWS, 15 jan. 2006. Disponível em: <www.cbsnews.com/stories/ 2006/01/15/sunday/main1209916.shtml>. Acesso em: 13 mar. 2007.

BLAKESLEE, T. The right brain: a new understanding of the unconscious mind and its creative powers. London: The Macmillan Press, 1980.

BOGEN, J.E. Some educational implications of hemispheric specialization. In: WITTROCK, M.C. (Org.). The human brain. Prentice-Hall: Englewood Cliffs, 1977. p.133-53.

The other side of the brain: an appositional mind. In: ORNSTEIN, R.E. (Org.). The nature of human consciousness. San Francisco: W.H. Freeman and Company, 1973. p.101-25.

BROWNLEE, C. Buff and brainy: exercising the body can benefit the mind. Sci. News Online, 25 fev. 2006a. Disponível em: <http://www.sciencenews.org/articles/ 20060225/bob10.asp>. Acesso em: 1 jun. 2006a.

Eat smart: foods may affect the brain as well as the body. Sci. News Online, 4 mar. 2006b. Disponível em: <http://www.sciencenews.org/articles/20060304/ bob8.asp>. Acesso em: 1 jun. 2006b.

CAPACCHIONE, L. The power of your other hand: a course in channeling the inner wisdom of the right brain. Franklin Lakes: New Page Books, 2001.

CBS. Retraining the brain: doctors test drug-free methods to restore lost mental capabilities. CBS NEWS, 15 jan. 2006. Disponível em: <http://www.cbsnews.com/ stories/2006/01/15/sunday/main1209916.shtml>. Acesso em: 10 abr. 2007.

CHAFETZ, M.D. Smart for life: how to improve your brain power at any age. New York: Penguin, 1992.

COHEN, I.; GOLDSMITH, M. Hands on: how to use brain gym in the classroom. Ventura, CA: Edu Kinesthetics, 2002.

COTMAN, C.W.; BERCHTOLD, N. C. Exercise: a behavioral intervention to enhance brain health and plasticity. Trends Neurosci., v.25, n.6, p.295-301, 2002.

DEAN, M. Governmentality: power and rule in modern society. London: Sage, 1999.

DENNISON, P.E.; DENNISON, G.E. Brain gym (teachers edition). Ventura, CA: EduKinesthetics, 1989.

DENNISON, G.E.; DENNISON, P.E.; TEPLITZ, J. V. Brain gym for business: instant brain boosters for on-the-job success. Ventura, CA: Edu-Kinesthetics, 1994.

DUMIT, J. Picturing personhood: brain scans and biomedical identity. Princeton: Princeton University Press, 2004.

EDWARDS, B. Drawing on the right side of the brain. Los Angeles: J. P. Tarcher, 1979. EHRENBERG, A. Le sujet cerebral. Esprit, n.309, p.130-55, 2004.

EHRENWALD, J. Anatomy of genius: split brains and global minds. New York: Human Sciences Press, 1984.

EIFFERT, S.D. Cross-train your brain: a mental fitness program for maximizing creativity and achieving success. New York: Amacom, 1999

FOUCAULT, M. Les anormaux. Cours au Collège de France, 1974-1975. Paris: Seuil/ Gallimard, 1999.

. Il faut défendre la sociéte. Cours au Collège de France 1975-1976. Paris:

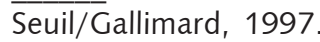


FOUCAULT, M. L'Usage des plaisirs. Paris: Gallimard, 1984a.

Le souci de soi. Paris: Gallimard, 1984b.

La volonté de savoir. Paris: Gallimard, 1976.

FRANZ, S.I. Variations in distribution of the motor centers. Psychol. Rev., supl.19, p.80162, 1915

FULLER, R.C. Spiritual, but not religious: understanding unchurched America. New York: Oxford University Press, 2001.

GAINER, R.S.; GAINER, H. Educating both halves of the brain: fact or fancy?. Art Educ., V.30, n.5, p.20-2, 1977.

GAZZANIGA, M.S. The ethical brain. New York: Dana Press, 2005.

$\overline{1985 .}$

The social brain: discovering the networks of the mind. New York: Basic Books,

The split brain in man. In: ORNSTEIN, R.E. (Org.). The nature of human consciousness. San Francisco: W.H. Freeman and Company, 1973. p.89-100.

GAZZANIGA, M.S.; BOGEN, J.E.; SPERRY, R.W. Some functional effects of sectioning the cerebral commissures in man. Proc. Nact. Acad. Sci., v.48, p.1765-9, 1962.

GIBBON, S.; NOVAS, C. (Orgs.). Biosocialities, genetics and the social sciences: making biologies and identities. New York: Routledge, 2008.

GOLDBERG, E. The executive brain: frontal lobes and the civilized mind. Oxford: Oxford University Press, 2001.

GOLDMAN, R.; KLATZ, R.; BERGER, L. Brain fitness (anti-aging strategies for achieving super mind power). New York: Doubleday, 1991.

GRAHAM BROWN, T.; SHERRINGTON, C.S. On the instability of a cortical point. Proc. R. Soc. Lond. B, v.85, n.579, p.250-77, 1912.

GREEN, M. Prophets of a new age: the politics of hope from the eighteenth through the twenty-first centuries. New York: Scribner, 1992.

GUNSFIELD, J.R. Nature's body and the metaphors of food. In: LAMONT, M.; FOURNIER, M. (Orgs.). Cultivating differences: symbolic boundaries and the making of inequality. Chicago: The University of Chicago Press, 1992. p.75-103.

HARRINGTON, A. Mind, medicine, and the double brain: a study in nineteenthcentury thought. Princeton: Princeton University Press, 1987.

HARRIS, L.J. Teaching the right brain: historical perspective on a contemporary educational fad. In: BEST, C.T. (Org.). Hemispheric function and collaboration in the child. New York: Academic Press, 1985. p.231-74.

Left-handedness: early theories, facts, and fancies. In: HERRON, J. (Org.). Neuropsychology of left-handedness. New York: Academic Press, 1980. p.3-78.

HEALY, D. The creation of psychopharmacology. Cambridge: Harvard University Press, 2002.

HERMANN, N. The creative brain. Train. Dev. J., n.35, p.11-6, 1981.

HUNTER, M. Right-brained kids in left-brained schools. Today's Educ., v.65, n.4, p.458, 1976.

ILLES, J.; RACINE, E. Neuroethics: dialogue on a continuum from tradition to innovation. Am. J. Bioeth., v.5, n.2, p.W3-W4, 2005.

JAMES, W. The principles of Psychology. Chicago, London, Toronto: Encyclopaedia Britannica, 1952. 
JONES, E.G. Plasticity and neuroplasticity. J. Hist. Neurosci., v.9, n.1, p.37-9, 2000.

KELLOG, J.H. First book in Physiology and hygiene. New York, Cincinnati, Chicago: Harper \& Brothers, 1887.

LASHEY, K. Temporal variation in the function of the gyrus precentralis in primates. Am. J. Physiol., n.65, p.585-602, 1923.

LAWTON, G. A game to train your brain? New Sci. Mag., n.2638, p.26-9, 2008.

LYNCH, Z. Neurotechnology and society (2010-2060). Ann. N. Y. Acad. Sci., v.1013, p.229-33, 2004.

MAASEN, S. et al. Self-help: the making of neosocial selves in neoliberal society. In: MAASEN, S.; SUTTER, B. (Orgs.). On willing selves: neoliberal politics vis-à-vis the neuroscientific challenge. Hampshire, New York: Palgrave MacMillan, 2007. p.25-52.

MALTZ, M. Psycho-cybernetics. New York: Prentice-Hall, 1960.

MARK, V.H.; MARK, J.P. Brain power: a neurosurgeon's complete program to maintain and enhance brain fitness throughout your life. Boston: Houghton Mifflin, 1991.

McGEE, M. Self-help, inc: makeover culture in American life. New York: Oxford University Press, 2005.

MERZENICH, M.M.; JENKINS, W.M. Cortical representations of learned behaviors. In: ANDERESEN, P. et al. (Orgs.). Memory concepts. New York: Elsevier, 1994. p.437-54.

OLNEY, J. Exercise may be key to keeping your brain fit. Abc7news.com. 24 fev. 2006. Disponível em: <www.abclocal.go.com $/ \mathrm{kgo/}$

story?section=assignment_7\&id=3939370 > . Acesso em: 5 jun. 2008.

ORNSTEIN, R.E. The right mind: making sense of the hemispheres. New York: Harcourt Brace and Company, 1997. 1972.

The Psychology of consciousness. New York: Harcourt, Brace, Jovanovich,

ORTEGA, F. O corpo incerto: corporeidade, tecnologias médicas e cultura contemporânea. Rio de Janeiro: Garamond, 2008.

ORTEGA, F.; VIDAL, F. Mapping the cerebral subject in contemporary culture. RECIIS Electron. J. Communic. Inform. Innovat. Health, v.1, n.2, p.255-9, 2007.

PARLETTE, S. The brain workout book. New York: M. Evans and Company, 1997.

RABINOW, P. Artificiality and enlightenment: from sociobiology to biossociality. In: Essays on the anthropology of reason. Princenton: Princenton University Press, 1996. p.91-111.

RABINOW, P.; ROSE, N. Biopower today. BioSocieties, n.1, p.195-217, 2006.

RACINE, E.; BAR-ILAN, O.; ILLES, J. FMRI in the public eye. Nat.Rev. Neurosci., v.6, n.2, p.159-64, 2005.

ROSE, N. The politics of life itself: biomedicine, power, and subjectivity in the twentyfirst century. Princeton: Princeton University Press, 2007.

RÜDIGER, F. Literatura de auto-ajuda e individualismo. Porto Alegre: Editora da Universidade/UFRGS, 1995.

SCHWARTZ, J.M.; BEGLEY, S. The mind \& the brain: neuroplasticity and the power of mental force. New York: HarperCollins, 2002.

SINGER, E. Exercising the brain: innovative training software could turn back the clock on aging brains. Technol. Rev., nov. 21, 2005. Disponível em: <http://

www.technologyreview.com/InfoTech-Software/wtr_15914,300,p1.html>. Acesso em: 1 jun. 2006. 
SPOTTS, D.; ATKINS, N. Super brain power: 28 minutes to a supercharged brain. Seattle: LifeQuest Publishing Group, 1999.

TANNEN, S. Mental fitness - exercises for the brain. 2005. Disponível em: < http:// www.bellydoc.com/articles/article7.htm >. Acesso em: 1 abr. 2008.

TEICHERT, N.W. The brain fitness exercises being tested improve seniors' mental abilities. 2005. Disponível em: <www.sacbee.com/content/news/story/13790575p14632268c.html >. Acesso em: 1 jun. 2006.

VIDAL, F. Brainhood, anthropological figure of modernity. Hist. Hum. Sci., v.22, n.1, p.5-36, 2009.

VRECKO, S. Folk neurology and the remaking of identity. Mol. Intervent., v.6, n.6, p.300-3, 2006.

WEILLER, C.; RIJNTJES, M. Learning, plasticity, and recovery in the central nervous system. Exp. Brain Res., v.128, n.1-2, p.134-8, 1999.

WELLS, C.G. Right brain sex: using creative visualization to enhance sexual pleasure. New York: Simon \& Schuster, 1989.

WINTER, A.; WINTER, R. Build your brain power: the latest techniques to preserve, restore, and improve your brain's potential. Nova York: St. Martin's Press, 1987.

WOLBRING, G. Neurodiversity, Neuroenhancement, Neurodisease, and Neurobusiness. Innov. Watch, 15 mai, 2007. Disponível em: <www.innovationwatch.com/ choiceisyours/choiceisyours-2007-04-30.htm>. Acesso em: 15 fev. 2008.

ORTEGA, F. Neurociencias, neuro-cultura y auto-ayuda cerebral. Interface - Comunic., Saude, Educ., v.13, n.31, p.247-60, out./dez. 2009.

Este artículo pretende analizar el fenómeno denominado neuro-ascesis o auto-ayuda cerebral en el contexto del creciente impacto de las neurociencias y del aparecimiento de la neuro-cultura y del sujeto creberal. Para tanto, es importante comprender el ámbito socio-cultural más amplio en el que la neuro-ascesis se inserta y que corresponde a lo que se viene llamando "cultura somática" o, más específicamente, bio-sociabilidad. El objeto del artículo es el de explorar como una forma de subjetividad reduccionista, el sujeto cerebral, da lugar a la aparición de prácticas de sí cerebrales; esto es, prácticas de como actuar sobre el cerebro para maximizar su actuación, que llevan a la formación de nuevas formas de sociabilidad.

Palabras clave: Neurociencias. Sujeto cerebral. Neuro-cultura. Auto-ayuda cerebral. 